\title{
HLA-DPA1 gene is a potential predictor with prognostic values in multiple myeloma
}

\author{
Jie Yang, Fei Wang and Baoan Chen ${ }^{*}$
}

\begin{abstract}
Background: Multiple myeloma (MM) is an incurable hematological tumor, which is closely related to hypoxic bone marrow microenvironment. However, the underlying mechanisms are still far from fully understood. We took integrated bioinformatics analysis with expression profile GSE110113 downloaded from National Center for Biotechnology Information-Gene Expression Omnibus (NCBI-GEO) database, and screened out major histocompatibility complex, class II, DP alpha 1 (HLA-DPA1) as a hub gene related to hypoxia in MM.

Methods: Differentially expressed genes (DEGs) were filtrated with R package "limma". Gene Ontology (GO) enrichment and Kyoto Encyclopedia of Genes and Genomes (KEGG) pathway were performed using "clusterProfiler" package in $R$. Then, protein-protein interaction (PPI) network was established. Hub genes were screened out according to Maximal Clique Centrality (MCC). PrognoScan evaluated all the significant hub genes for survival analysis. ScanGEO was used for visualization of gene expression in different clinical studies. $P$ and Cox $p$ value $<0.05$ was considered to be statistical significance.
\end{abstract}

Results: HLA-DPA1 was finally picked out as a hub gene in MM related to hypoxia. MM patients with down-regulated expression of HLA-DPA1 has statistically significantly shorter disease specific survival (DSS) (COX $p=0.005411$ ). Based on the clinical data of GSE47552 dataset, HLA-DPA1 expression showed significantly lower in MM patients than that in healthy donors (HDs) $(p=0.017)$.

Conclusion: We identified HLA-DPA1 as a hub gene in MM related to hypoxia. HLA-DPA1 down-regulated expression was associated with MM patients' poor outcome. Further functional and mechanistic studies are need to investigate HLADPA1 as potential therapeutic target.

Keywords: Multiple myeloma, Hypoxia, Prognosis, Bioinformatics analysis

\section{Background}

Multiple myeloma (MM) is a hematological malignancy which is characterized by aberrant plasma cells infiltration in the bone marrow and complex heterogeneous cytogenetic abnormalities [1]. Accumulation of abnormal plasma cells replaces normal hematopoietic cells and

\footnotetext{
*Correspondence: cba8888@hotmail.com

Department of Hematology and Oncology, Zhongda Hospital, School of Medicine, Southeast University, No. 87, Dingjiaqiao, Gulou District, Nanjing 210009, Jiangsu, China
}

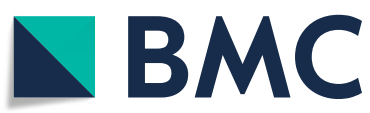

(C) The Author(s). 2020 Open Access This article is licensed under a Creative Commons Attribution 4.0 International License, which permits use, sharing, adaptation, distribution and reproduction in any medium or format, as long as you give appropriate credit to the original author(s) and the source, provide a link to the Creative Commons licence, and indicate if changes were made. The images or other third party material in this article are included in the article's Creative Commons licence, unless indicated otherwise in a credit line to the material. If material is not included in the article's Creative Commons licence and your intended use is not permitted by statutory regulation or exceeds the permitted use, you will need to obtain permission directly from the copyright holder. To view a copy of this licence, visit http://creativecommons.org/licenses/by/4.0/. The Creative Commons Public Domain Dedication waiver (http://creativecommons.org/publicdomain/zero/1.0/) applies to the data made available in this article, unless otherwise stated in a credit line to the data. leads to "CRAB" - hypercalcemia, renal failure, anemia, and bone lesions, even fetal outcome eventually [2]. With the deepening of basic and clinicalresearches, novel drugs mainly including proteasome inhibitors and immunomodulatory drugs have improved patients' outcome to some extend [3, 4]. Besides, high-dose chemotherapy and tandem autologous stem cell transplant (ASCT), together with supportive care have significantly prolonged patients' progression-free survival (PFS) and overall survival (OS) [5]. However, MM remains an 
uncurable disease as underlying molecular mechanisms of pathogenesis and progression are still largely unclear. Quite a few patients cannot get diagnosis and proper treatment in time. Therefore, identifying key mechanisms regulating $\mathrm{MM}$ is critically important for early diagnosis and targeted therapy.

With the advances of high-throughput platforms and microarray, more and more molecular heterogeneity on MM has been recognized [6, 7]. Hypoxia plays an important role in occurrence and development of MM $[8,9]$ and more related pathogenesis is still urgent needs to be explore for better diagnosis and treatment. In order to find potential biomarker of MM related to hypoxia, we analyzed the differentially expressed genes (DEGs) functions and pathways between normoxic and hypoxia-resistant (HR) MM cell lines contained in GSE110113 dataset. Major histocompatibility complex, class II, DP alpha 1 (HLA-DPA1) was finally screened out as a hub gene associated with poor outcome of MM related to hypoxia. In addition, survival analyses and gene expression level were visualized with online clinical data, and the results validated higher HLA-DPA1expression level of MM patients was associated with poor clinical outcome. The findings in this study provide new insights on HLA-DPA1 as a potential biomarker for MM and more research needs to be performed.

\section{Methods}

\section{Data source and DEGs identification}

Gene expression profile GSE110113 was downloaded from National Center for Biotechnology InformationGene Expression Omnibus (NCBI-GEO) database (https://www.ncbi.nlm.nih.gov/geo/) [10]. The array data of GSE110113 were generated on GPL6244 platform (HuGene-1_0-st Affymetrix Human Gene 1.0 ST Array). There are four parental cells (RPMI8226, KMS-11, U266, IM-9) and four HR cells that derived from above parental cells. Two group cells were cultured under normoxic condition $\left(20 \% \mathrm{O}_{2}\right)$ and hypoxic condition $(1 \%$ $\mathrm{O}_{2}$ ) for $24 \mathrm{~h}$, respectively.

$\mathrm{R}$ package "limma" was used to identify DEGs between normoxic and HR cells groups [11]. The screen criteria were adjusted $p$ value $<0.05$ and $[\log 2$ FoldChange $(\log 2 \mathrm{FC})]>1$. All genes were visualized by volcanic maps and top 50 dramatically altered genes were selected to draw a heatmap by $R$ package "ggplot2" [12].

\section{GO and KEGG analysis}

Gene Ontology (GO) enrichment analysis and Kyoto Encyclopedia of Genes and Genomes (KEGG) pathway analysis were conducted by using $\mathrm{R}$ package "clusterProfiler" [13] which is for functional classification and gene clusters enrichment. GO enrichment includes biological process (BP), molecular function (MF), and cellular component $(\mathrm{CC})$ three subontologies. Analysis results were displayed with "GOplot" package of R [14]. In addition, relationship between pathways was further analyzed with the ClueGO plug-ins of Cytoscape software 3.7.2 [15]. A $p$ value less than 0.05 was considered statistically significant.

\section{PPI network analysis}

To clarify the relationships among proteins encoded by selected enrichment genes, a protein-protein interaction (PPI) network was established using the STRING database (https://string-db.org) [16]. Cytoscape software 3.7.2 was used to visualize the genes with minimum interaction score more than 0.4 [15]. Then, we utilized cytoHubba plug-ins to recognize interaction degree of hub-gene clustering according to the Maximal Clique Centrality (MCC) methods. Wayne diagram produced by online tool Bioinformatics \& Evolutionary Genomics (http://bioinformatics.psb.ugent.be/webtools/Venn/) was used to show the overlapped genes.

\section{Survival analysis}

To assess the prognostic value of selected genes in MM patients, survival analysis was performed with the PrognoScan database (http://dna00.bio.kyutech.ac.jp/PrognoScan/) [17]. PrognoScan explores the relationship between gene expression and prognosis of patients, across all the public available microarray datasets provided. The results are displayed with hazard ratio (HR) and Cox $p$ value from a Log-rank test. Cox $p$ value $<0.05$ was considered statistically significant. Based on GSE2658 dataset $(n=559)$ provided by Zhan [18], relationship between gene expression and corresponding disease specific survival (DSS) were researched. Besides, according to online ScanGEO database (http://scangeo. dartmouth.edu/ScanGEO/) [19], we chose $p$ value $<0.05$ as significance criterion and screened out GSE47552 [20] and GSE2113 [21] datasets which involved HLA-DPA1 expression level compared to different degree of disease progression and healthy donors (HDs). Gene expression level in clinical patients was explored with the two datasets.

\section{Results \\ Identification of DEGs}

This study was performed as a multiple strategy to pick out the hub gene related to hypoxia in MM dataset GSE110113. The hub gene was then validated with online clinical data (Fig. 1). Myeloma cells were divided into normoxic and HR groups. Totally, 1285 DEGs were identified including 614 up-regulated and 671 downregulated genes using "limma" $\mathrm{R}$ package (Fig. 2a) and a heatmap depicted top 50 genes (Fig. $2 \mathrm{~b}$ ). 


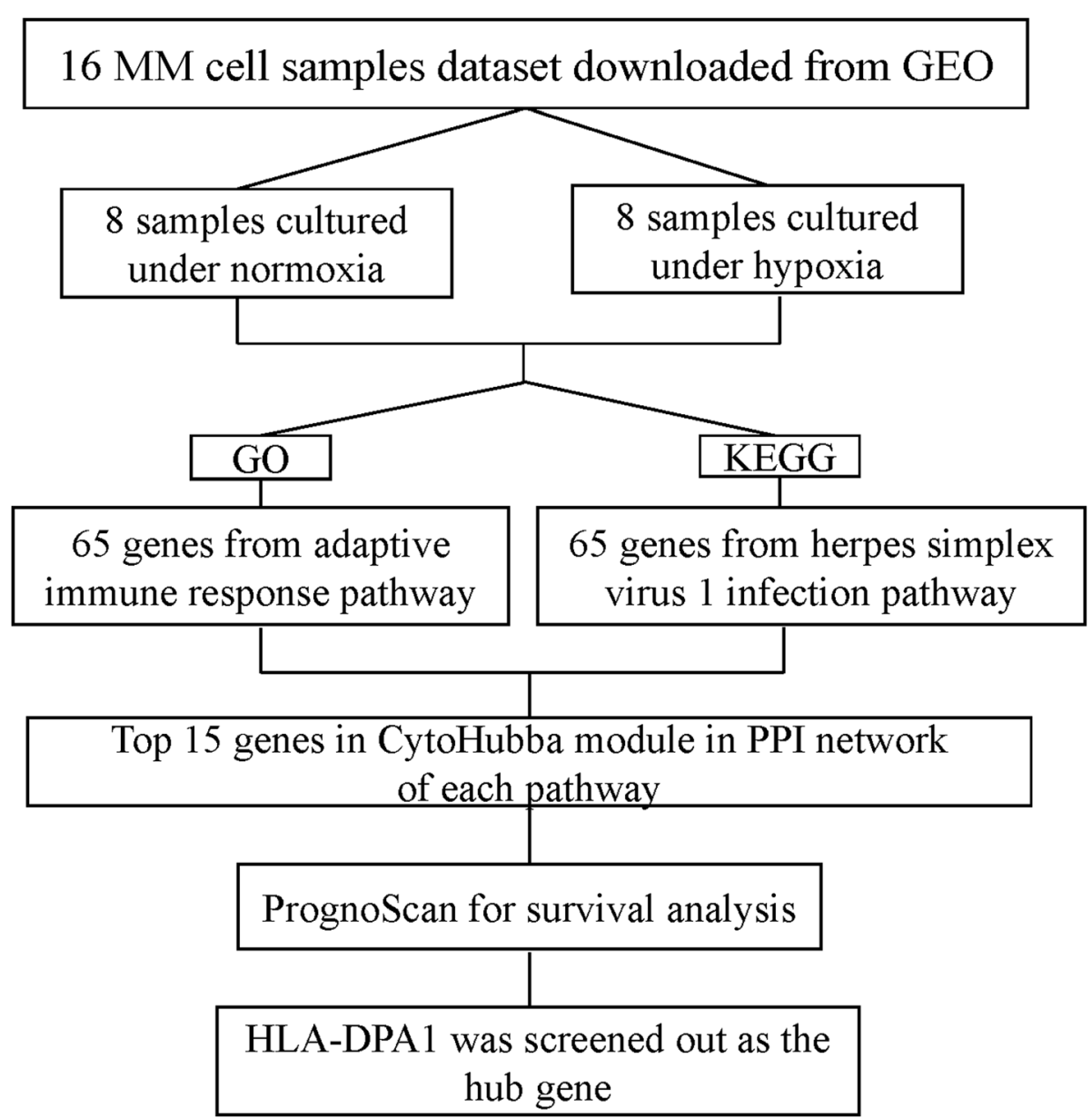

Fig. 1 A schematic view of the procedure of the study with GSE110113

\section{GO and KEGG enrichment analysis}

GO and KEGG enrichment analyses were performed with all DEGs to further explore their functions with $\mathrm{R}$ package "clusterProfiler". Three subontologies including $\mathrm{BP}, \mathrm{MF}$, and $\mathrm{CC}$ were examined in GO analysis. Adaptive immune response pathway $(p=1.31 \mathrm{e}-10$, FDR $=$ $6.59 \mathrm{e}-07)$, cell adhesion molecule binding pathway ( $p=$ $0.000162, \mathrm{FDR}=0.104$ ) and receptor complex pathway $(p=1.23 \mathrm{e}-05, \mathrm{FDR}=0.00221)$ were selected as the most significant pathway in each subontologies, respectively (Fig. 3a-c). According to their $p$ values, we selected adaptive immune response for further analysis and found 65 DEGs was enriched in this GO term. The top enriched pathway of the DEGs in KEGG enrichment analysis was herpes simplex virus 1 infection pathway $(p=1.39 \mathrm{e}-08, \mathrm{FDR}=3.63 \mathrm{e}-06)$ (Fig. 3d). We further used ClueGO to analyze and show the interrelation of the enriched pathways and the DEGs. Herpes simplex virus 1 infection pathway remained the most significant pathway, and there were 70 DEGs involved in this pathway (Figs. 3e, f).
Totally, 65 and 70 DEGs were involved in the two selected pathways, respectively (Table 1). Next, we identified 9 common genes by overlapping DEGs in the two selected pathways with Wayne diagram (Fig. 3g). They were SYK, POU2F2, LTA, HLA-DPB1, HLA-DQA1, HLA-DQB1, HLA-DPA1, HLA-DMA and HLA-DMB.

\section{PPI network}

To pick out and further understand the hub genes, we firstly constructed the PPI network consisting of all the DEGs from the two most significant pathways mentioned above in STRING (Fig. 4a, b), respectively. Then, we used Cytoscape plug-ins cytoHubba to screen top 15 candidate hub genes of each pathway according nodes rank (Fig. 4c, d) and they are listed in Table 2. Subsequently, we identified 3 common genes in the two sets of top 15 hub genes, including HLA-DPA1, DQHLA-DQA1 and HLA-DQB1 as candidate hub genes. 


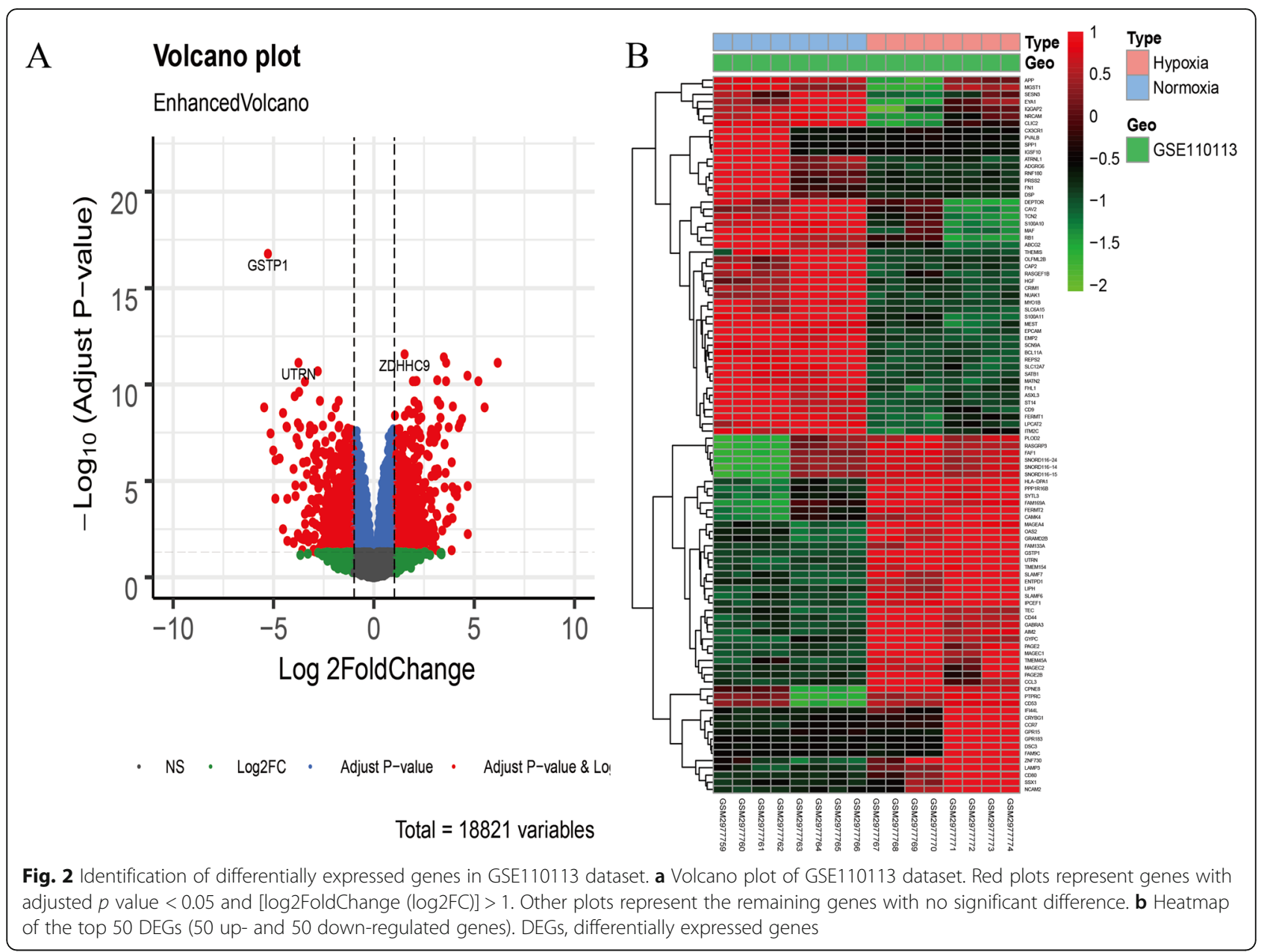

\section{Survival analysis}

Finally, we evaluated the correlation between candidate hub genes and the prognosis of patients with MM. Potential prognostic value of the candidate hub genes were assessed with PrognoScan. The result showed that only HLA-DPA1 (Cox $p=0.005411$ ) was statistically significant associated with DSS of MM patients based on 559 patients in GSE2658 dataset (Fig. 5a, Additional file 1). In addition, ScanGEO exploration results showed expression level of HLADPA1 in MM patients was significant lower than that in HDs $(p=0.017)$ according to GSE47552 dataset (Fig. 5b). The clinical characteristics of the MM patients [20] in GSE47552 dataset is showed in Additional file 2. Regarding GSE2113, there are 7 monoclonal gammopathy of undetermined significance (MGUS), 39 newly diagnosed MM and 6 plasma-cell leukemia (PCL) patients. As the severity of the disease woresned, the level of HLA-DPA1 gene expression gradually decreased ( $p=0.007$ ) (Fig. 5c). Further verification of this gene in clinical research remains need.

\section{Discussion}

In this study, we analyzed 1285 DEGs between normoxic and hypoxic cultured MM cells based on GSE110113 dataset. Enrichment analysis indicated that adaptive immune response was the most significant GO term and herpes simplex virus 1 infection pathway was the most significant KEEG pathway. It is well-known that human immune system can eradicate cancer cells. Cancers' occurrence and development is critically associated with immune response adaptation and immune escape which have been demonstrated with mice model [22, 23]. Herpes simplex virus (HSV) 1 has antitumor effect which mainly depends on its cytotoxic effect and replication ability with tumor in order to produce more virus for tumor lysis [24]. Previous study indicated HSV was associated with occurrence of MM and Bortezomib could inhibit HSV infection by halting viral capsid transport to the nucleus [25].

Establishment of the PPI network and further analysis with Cytoscape plug-ins cytoHubba identified 3 candidate hub genes, HLA-DPA1, HLA-DQA1 and HLA- 


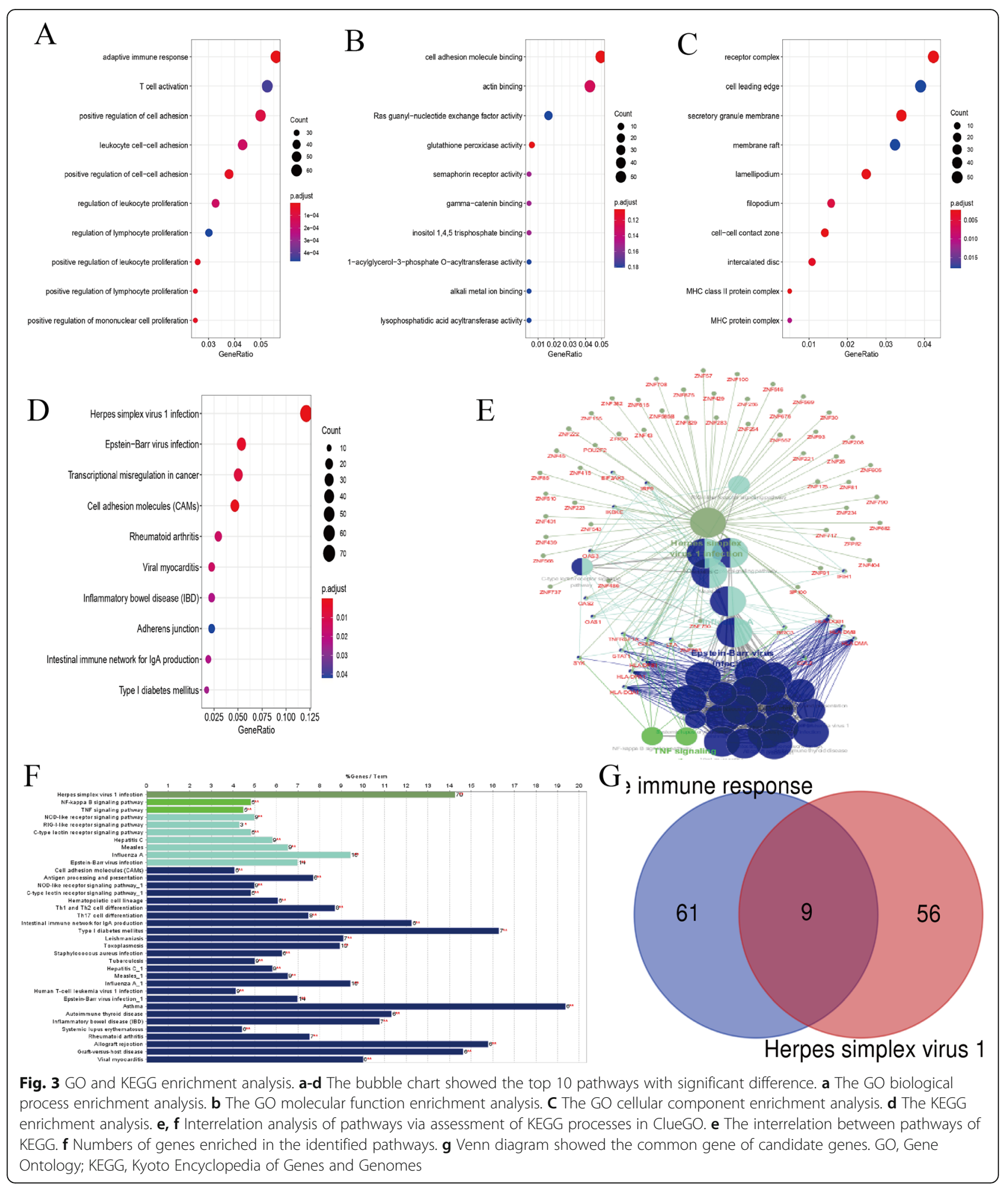

DQB1. The major histocompatibility complex (MHC) class II proteins include HLA-DR, HLA-DQ and HLADP classical proteins and they only expressed on professional antigen-presenting cells (B lymphocytes, dendritic cells and macrophages) to activate CD4+ $\mathrm{T}$ cells [26].
They could participate in cancer development as it has been proved that dysregulation of immune function which involved antigen presentation was associated with cancer [27]. Subsequently, survival analysis based on GSE2658 dataset with PrognoScan revealed HLA-DPA1 
Table 1 DEGs identified from selected pathways of GO and KEGG

\begin{tabular}{ll}
\hline DEGs & Gene names \\
\hline Adaptive immune response & ADA, ADCY7, CD8B, DENND1B, EMP2, FAM49B, IGKV1D-8, LAIR1, PYCARD, SMAD7, SYK, THEMIS, TLR4, TNFRSF1B, \\
pathway & TNFRSF21, ULBP3, UNC93B1, ZP3, BATF, C2, CAMK4, CD274, CD48, CD70, CD79A, CD79B, CD80, CD86, CEACAM1, \\
& CTSH, ERAP2, GPR183, HAVCR2, HLA-DMA, HLA-DMB, HLA-DPA1, HLA-DPB1, HLA- DQA1, HLA-DQB1, ICAM1, IL23A, \\
& IL23R, INPP5D, JAK3, LAMP3, LILRB4, LTA, MEF2C, NFKBIZ, PAG1, POU2F2, PTPRC, RAB27A, RORA, SAMSN1, SASH3, \\
& SLAMF1, SLAMF6, SLAMF7, SPN, TEC, TFRC, TNFAIP3, TNFSF13B, TXK \\
Herpes simplex virus 1 & CCL2, IKBKE, SYK, TNFRSF1A, ZNF26, ZNF382, ZNF605, ZNF717, BIRC3, CHUK, EIF2AK3, HLA-DMA, HLA-DMB, HLA-DPA1, \\
infection pathway & HLA-DPB1, HLA-DQA1, HLA-DQB1, IFIH1, IRF9, LTA, OAS1, OAS2, OAS3, POU2F2, SP100, STAT1, ZFP30, ZFP82, ZNF100, \\
& ZNF155, ZNF175, ZNF208, ZNF221, ZNF222, ZNF223, ZNF234, ZNF254, ZNF256, ZNF283, ZNF30, ZNF404, ZNF415, \\
& ZNF429, ZNF43, ZNF431, ZNF439, ZNF45, ZNF486, ZNF510, ZNF543, ZNF546
\end{tabular}

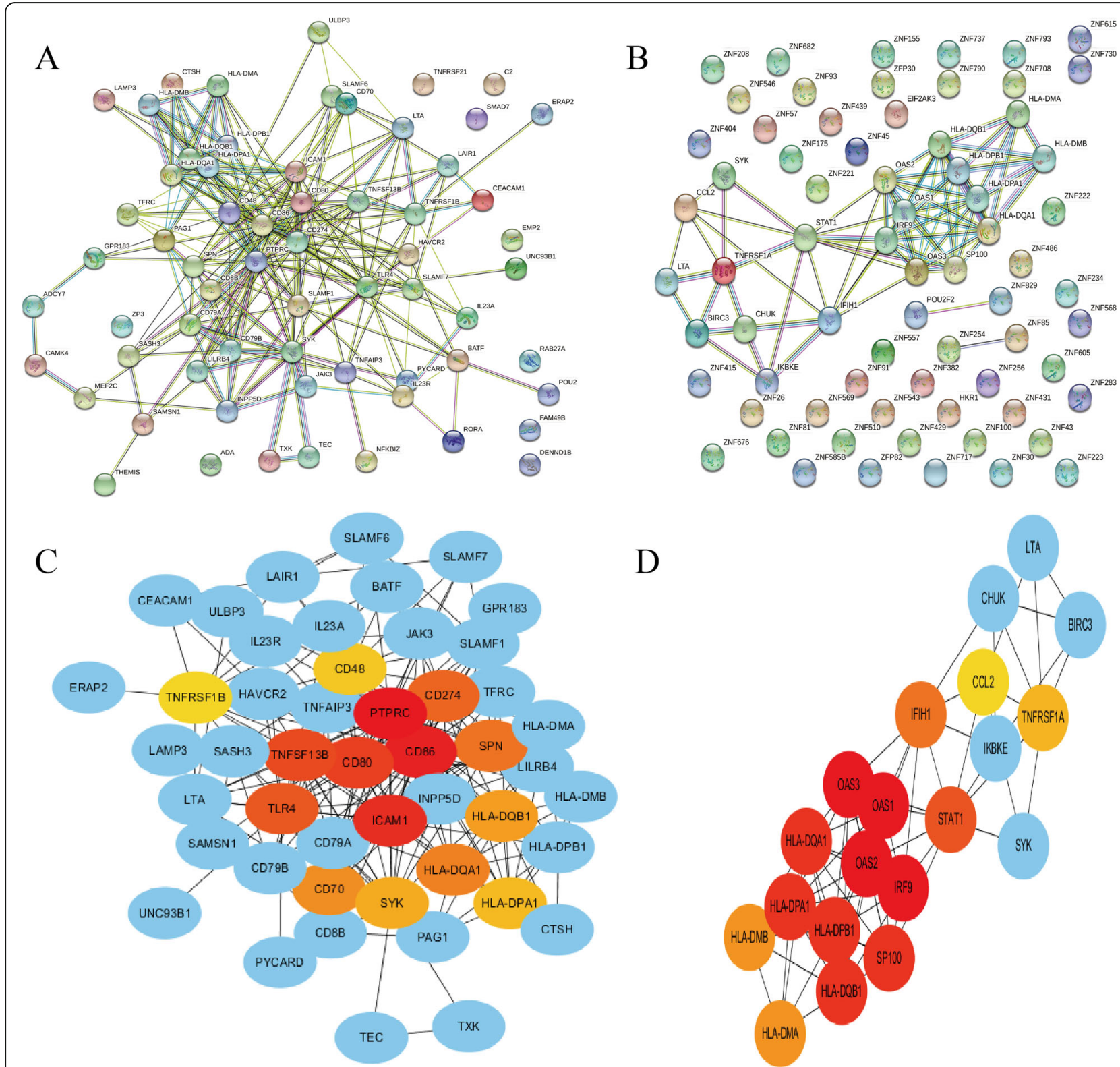

Fig. 4 PPI network analysis. a, b The PPI analysis at STRING. c, d Cytoscape plug-ins cytoHubba analysis of candidate genes after PPI analysis. a, c Genes identified from adaptive immune response pathway. b, d Genes identified from herpes simplex virus 1 infection pathway. PPI, protein-protein interaction 
Table 2 The top 15 genes with the highest score of each pathway through the Cytoscape "cytoHubba" module analysis

\begin{tabular}{|c|c|c|c|c|}
\hline \multirow{2}{*}{$\begin{array}{l}\text { Top } 15 \\
\text { Rank }\end{array}$} & \multicolumn{2}{|c|}{ Adaptive immune response pathway } & \multicolumn{2}{|c|}{ Herpes simplex virus 1 infection pathway } \\
\hline & Name & Score & Name & Score \\
\hline 1 & PTPRC & 11,394 & IRF9 & 40,560 \\
\hline 2 & CD86 & 9512 & OAS1 & 40,560 \\
\hline 3 & ICAM1 & 9390 & OAS2 & 40,560 \\
\hline 4 & CD80 & 9146 & OAS3 & 40,560 \\
\hline 5 & TNFSF13B & 5760 & SP100 & 40,440 \\
\hline 6 & TLR4 & 5337 & HLA-DQB1 & 40,440 \\
\hline 7 & CD274 & 4108 & HLA-DQA1 & 40,440 \\
\hline 8 & SPN & 3648 & HLA-DPB1 & 40,440 \\
\hline 9 & HLA-DQA1 & 3528 & HLA-DPA1 & 40,440 \\
\hline 10 & CD70 & 2880 & STAT1 & 250 \\
\hline 11 & HLA-DQB1 & 2808 & $\mathrm{IF|H1}$ & 126 \\
\hline 12 & SYK & 2410 & HLA-DMB & 120 \\
\hline 13 & HLA-DPA1 & 1992 & HLA-DMA & 120 \\
\hline 14 & CD48 & 1566 & TNFRSF1A & 12 \\
\hline 15 & TNFRSF1B & 1493 & CCL2 & 10 \\
\hline
\end{tabular}

as the hub gene associated with DSS of MM patients. Since GSE2658 dataset did not provided detail clinical data of patients' general condition, multivariate Cox's proportional hazard regression models could not be constructed to further clarify the relationship between HLADPA1 and survival. According to ScanGEO analysis results, gene expression of HLA-DPA1 was significantly lower compared to HDs and MGUS.

Hypoxia is common and essential in various cancers which can bring different gene expression change during metabolic adaptations [28]. As a result, cancer cells can survival and keep high rate proliferation. Previous studies have shown hypoxic bone marrow microenvironment plays a critical role in MM occurrence and progression through different aspects. For instance, endothelial cells (ECs) in MM patients having a hypoxic phenotype could keep up with enhanced angiogenesis in cancer growth and metastasis [8]. Hypoxia induced MM cells dedifferentiation, stem-cell like state acquisition without apoptosis and enhanced drug resistance to proteasome inhibitors [9].

In the GO enrichment analysis, cell adhesion molecule binding was the most significant term. Evidences suggested cell adhesion molecule binding is an important pathway in MM related to hypoxia. Hypoxia reduces the adhesion of tumor cells and accelerates tumor development process [7, 29], manifested as extramedullary (EMD). Central nervous system (CNS) involvement phenotype is an rare, EMD form of MM which indicates unfavorable cytogenetics, shorter survival time even with intensive treatment [30]. Capicua transcriptional repressor (CIC) is a transducer of receptor tyrosine kinase (RTK) signaling that functions through default repression [31]. Marra MA et al. found that CIC deficiency was associated with down-regulated expression of genes involving in cellcell adhesion which led to tumor progression and overexpression mitogen-activated protein kinase (MAPK) signaling cascade [32]. Another research proved CIC mutation affected the BRAF-RAS pathway and resulted in drug resistance in MM patients [33]. Other several mutations including Ki-ras2 Kirsten rat sarcoma viral oncogene homolog (KRAS), Neuroblastoma Ras viral oncogene homolog (NRAS) also participate in drug resistance of MM [34, 35]. In our study, HLA-DPA1 is also downregulated under hypoxic condition and we hypothesize that it may play an oncogenic role in MM through hypoxic activated signaling pathway.

HLA-DPA1, also known as HLA-DP1A, HLASB or DPA1, belongs to the HLA class II alpha chain paralogues [36]. As a result, HLA-DPA1 function as an MHC class II receptor to participate in immune response and antigenic peptides presentation. Clinical study on adrenocortical tumors (ACT) indicated low expression of HLA-DPA1 was associated with poor prognosis [37]. Acute myeloid leukemia (AML) relapse after transplantation was analyzed by Christopher MJ et al. It was proved to be associated with dysregulation of pathways which had an influence on immune function. HLA-DPA1 and several other MHC class II genes' down-regulation were involved as they function in antigen presentation [38]. Other several researches showed MHC class II genes had crucial relationship with cancer immunology, and down-regulation of related genes indicated a poor prognosis [26, 39, 40]. 
a

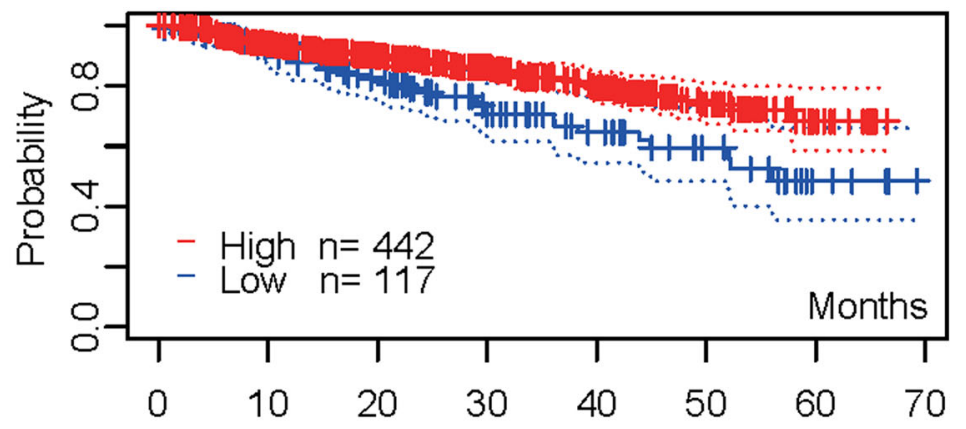

b

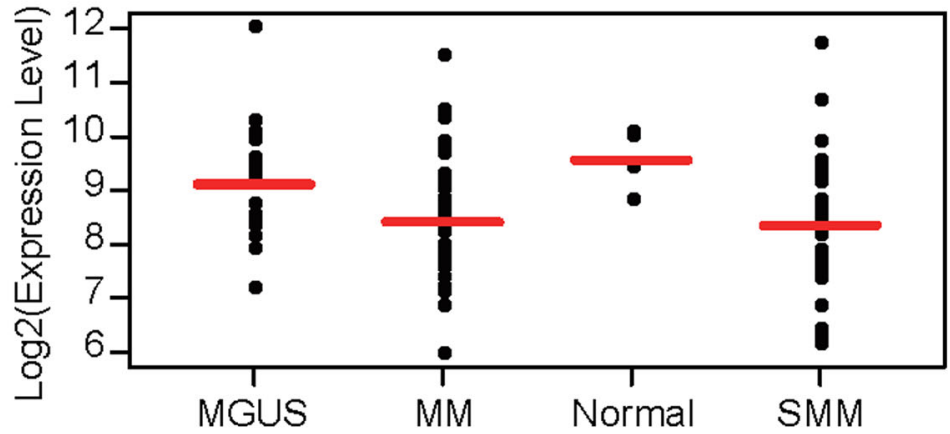

$\mathrm{C}$

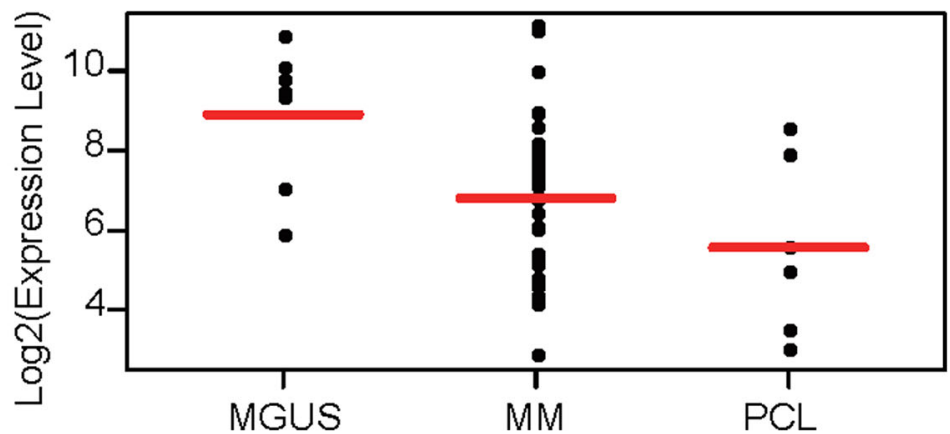

Fig. 5 Analysis of hub gene HLA-DPA1. a Kaplan-Meier survival curves comparing high and low expression of HLA-DPA1 in MM with PrognoScan (Cox $p=0.005411)$. b, $\mathbf{c}$ HLA-DPA1 gene expression in different clinical datasets. b HLA-DPA1 gene expression in GSE47552 dataset ( $p=0.017)$. $\mathbf{c}$ HLA-DPA1 gene expression in GSE2113 dataset $(p=0.007)$. MGUS, monoclonal gammopathy of undetermined significance; MM, multiple myeloma; SMM, smoldering multiple myeloma; $\mathrm{PCL}$, plasma-cell leukemia

\section{Conclusion}

HLA-DPA1 was a hub gene related to hypoxia in MM. Down-regulated expression of HLA-DPA1 was associated with shorter survival time of MM patients. Notably, 3 candidate hub genes were all related to immune response. Based on the findings in our study, further researches investigating immune process of MM pathogenesis may help us to better understand MM. This study provided a novel insight into HLA-DPA1 as a critical potential biomarker for MM.

\section{Supplementary information}

Supplementary information accompanies this paper at https://doi.org/10. 1186/s12885-020-07393-0.

Additional file 1.

Additional file 2.

\section{Abbreviations}

MM: Multiple myeloma; NCBI-GEO: National Center for Biotechnology Information-Gene Expression Omnibus; HLA-DPA1: Major histocompatibility complex, class II, DP alpha 1; DEGs: Differentially expressed genes; GO: Gene 
Ontology; KEGG: Kyoto Encyclopedia of Genes and Genomes; PPI: Proteinprotein interaction; MCC: Maximal Clique Centrality; HR: Hazard ratio; DSS: Disease specific survival; HDs: Healthy donors; ASCT: Autologous stem cell transplant; PFS: Progression-free survival; OS: Overall survival; HR: Hypoxia-resistant; log2FC: log2FoldChange; BP: Biological process; MF: Molecular function; CC: Cellular component; MGUS: Monoclonal gammopathy of undetermined significance; PCL: Plasma-cell leukemia; HSV: Herpes simplex virus; MHC: Major histocompatibility complex; ECs: Endothelial cells; EMD: Extramedullary; CNS: Central nervous system; CIC: Capicua transcriptional repressor; RTK: Receptor tyrosine kinase; MAPK: Mitogen-activated protein kinase; KRAS: Ki-ras2 Kirsten rat sarcoma viral oncogene homolog; NRAS: Neuroblastoma Ras viral oncogene homolog; ACT: Adrenocortical tumors; AML: Acute myeloid leukemia

\section{Acknowledgements}

Not applicable.

\section{Authors' contributions}

JY performed mainly data analysis and wrote the manuscript. FW performed part data analysis. B-A C conceived of and designed the study. All authors read and approved the final manuscript.

\section{Funding}

This study was supported by Natural Science Foundation of Jiangsu Province for Youth (BK20180372), Jiangsu Provincial Medical Youth Talent (QNRC2016812), and Key Medical of Jiangsu Province (ZDXKB2016020). The funders had no roles in study design, data collection, data analysis and interpretation, or writing of the manuscript.

\section{Availability of data and materials}

The dataset analysed during the current study are available in the NCBI-GEO repository, https://www.ncbi.nlm.nih.gov/geo/query/acc.cgi?acc=GSE110113.

\section{Ethics approval and consent to participate}

Not applicable.

\section{Consent for publication}

Not applicable.

\section{Competing interests}

The authors declare that they have no competing interests.

Received: 19 June 2020 Accepted: 9 September 2020

Published online: 24 September 2020

\section{References}

1. Hideshima T, Mitsiades C, Tonon G, Richardson PG, Anderson KC. Understanding multiple myeloma pathogenesis in the bone marrow to identify new therapeutic targets. Nat Rev Cancer. 2007;7(8):585-98.

2. Pawlyn C, Morgan GJ. Evolutionary biology of high-risk multiple myeloma. Nat Rev Cancer. 2017;17(9):543-56.

3. Röllig C, Knop S, Bornhäuser M. Multiple myeloma. Lancet. 2015;385(9983): 2197-208.

4. Pawlyn C, Davies FE. Toward personalized treatment in multiple myeloma based on molecular characteristics. Blood. 2019;133(7):660-75.

5. Sonneveld P, Avet-Loiseau H, Lonial S, Usmani S, Siegel D, Anderson KC, Chng WJ, Moreau P, Attal M, Kyle RA, et al. Treatment of multiple myeloma with high-risk cytogenetics: a consensus of the international myeloma working group. Blood. 2016;127(24):2955-62.

6. Long $\mathrm{S}$, Long $\mathrm{S}$, He $\mathrm{H}$, Chen $\mathrm{G}$. MicroRNA-765 is pregulated in multiple myeloma and serves an oncogenic role by directly targeting SOX6. Exp Ther Med. 2019;17(6):4741-7.

7. Zhu FX, He YC, Zhang JY, Wang HF, Zhong C, Wang XT. Using prognosisrelated gene expression signature and connectivity map for personalized drug repositioning in multiple myeloma. Med Sci Monit. 2019;25:3247-55.

8. Filippi I, Saltarella I, Aldinucci C, Carraro F, Ria R, Vacca A, Naldini A. Different adaptive responses to hypoxia in Normal and multiple myeloma endothelial cells. Cell Physiol Biochem. 2018;46(1):203-12.

9. Muz B, de la Puente P, Azab F, Luderer M, Azab AK. Hypoxia promotes stem cell-like phenotype in multiple myeloma cells. Blood Cancer J. 2014;4:e262.
10. Barrett T, Wilhite SE, Ledoux P, Evangelista C, Kim IF, Tomashevsky M, Marshall KA, Phillippy KH, Sherman PM, Holko M, et al. NCBI GEO: archive for functional genomics data sets--update. Nucleic Acids Res. 2013;41(Database issue):D991-5.

11. Ritchie ME, Phipson B, Wu D, Hu Y, Law CW, Shi W, Smyth GK. limma powers differential expression analyses for RNA-sequencing and microarray studies. Nucleic Acids Res. 2015;43(7):e47.

12. Ito K, Murphy D. Application of ggplot2 to Pharmacometric graphics. CPT Pharmacometrics Syst Pharmacol. 2013;2:e79.

13. Yu G, Wang LG, Han Y, He QY. clusterProfiler: an R package for comparing biological themes among gene clusters. OMICS. 2012;16(5):284-7.

14. Walter WFS-C, Ricote M. GOplot: An R Package for Visually Combining Expression Data With Functional Analysis. Bioinformatics. 2015;31(17):2912-4.

15. Shannon P, Markiel A, Ozier O, Baliga NS, Wang JT, Ramage D, Amin N, Schwikowski B, Ideker T. Cytoscape: a software environment for integrated models of biomolecular interaction networks. Genome Res. 2003;13(11):2498-504.

16. Szklarczyk D, Gable AL, Lyon D, Junge A, Wyder S, Huerta-Cepas J, Simonovic M, Doncheva NT, Morris JH, Bork P, et al. STRING v11: proteinprotein association networks with increased coverage, supporting functional discovery in genome-wide experimental datasets. Nucleic Acids Res. 2019; 47(D1):D607-13.

17. Mizuno H, Kitada K, Nakai K, Sarai A. PrognoScan: a new database for metaanalysis of the prognostic value of genes. BMC Med Genet. 2009;2:18.

18. Zhan F, Barlogie B, Arzoumanian V, Huang Y, Williams DR, Hollmig K, Pineda-Roman M, Tricot G, van Rhee F, Zangari M, et al. Gene-expression signature of benign monoclonal gammopathy evident in multiple myeloma is linked to good prognosis. Blood. 2007;109(4):1692-700.

19. Koeppen K, Stanton BA, Hampton TH, Wren J. ScanGEO: parallel mining of high-throughput gene expression data. Bioinformatics. 2017;33(21):3500-1.

20. Lopez-Corral L, Corchete LA, Sarasquete ME, Mateos MV, Garcia-Sanz R, Ferminan E, Lahuerta JJ, Blade J, Oriol A, Teruel Al, et al. Transcriptome analysis reveals molecular profiles associated with evolving steps of monoclonal gammopathies. Haematologica. 2014;99(8):1365-72.

21. Mattioli M, Agnelli L, Fabris S, Baldini L, Morabito F, Bicciato S, Verdelli D, Intini D, Nobili L, Cro L, et al. Gene expression profiling of plasma cell dyscrasias reveals molecular patterns associated with distinct IGH translocations in multiple myeloma. Oncogene. 2005;24(15):2461-73.

22. Ribas A. Adaptive immune resistance: how Cancer protects from immune attack. Cancer Discov. 2015;5(9):915-9.

23. Matsushita H, Vesely MD, Koboldt DC, Rickert CG, Uppaluri R, Magrini VJ, Arthur CD, White JM, Chen YS, Shea LK, et al. Cancer exome analysis reveals a T-cell-dependent mechanism of cancer immunoediting. Nature. 2012; 482(7385):400-4.

24. Stanziale SFPH, Adusumilli PS, Ben-Porat L, Gonen M, Fong Y. Infection with Oncolytic herpes simplex Virus-1 induces apoptosis in neighboring human Cancer cells a potential target to increase anticancer activity. Clin Cancer Re. 2004;10(9):3225-32.

25. Schneider SM, Pritchard SM, Wudiri GA, Trammell CE, Nicola AV. Early Steps in Herpes Simplex Virus Infection Blocked by a Proteasome Inhibitor. mBio. 2019;10(3):e00732-19.

26. Rimsza LM, Roberts RA, Miller TP, Unger JM, LeBlanc M, Braziel RM, Weisenberger DD, Chan WC, Muller-Hermelink HK, Jaffe ES, et al. Loss of MHC class II gene and protein expression in diffuse large B-cell lymphoma is related to decreased tumor immunosurveillance and poor patient survival regardless of other prognostic factors: a follow-up study from the leukemia and lymphoma molecular profiling project. Blood. 2004;103(11):4251-8.

27. Chamuleau ME, Ossenkoppele GJ, van de Loosdrecht AA. MHC class II molecules in tumour immunology: prognostic marker and target for immune modulation. Immunobiology. 2006;211(6-8):619-25.

28. Semenza GL. Hypoxia-inducible factors in physiology and medicine. Cell. 2012;148(3):399-408.

29. Viziteu E, Grandmougin C, Goldschmidt H, Seckinger A, Hose D, Klein B, Moreaux J. Chetomin, targeting HIF-1alpha/p300 complex, exhibits antitumour activity in multiple myeloma. Br J Cancer. 2016;114(5):519-23.

30. Jurczyszyn A, Grzasko N, Gozzetti A, Czepiel J, Cerase A, Hungria V, Crusoe E, Silva Dias AL, Vij R, Fiala MA, et al. Central nervous system involvement by multiple myeloma: a multi-institutional retrospective study of 172 patients in daily clinical practice. Am J Hematol. 2016;91(6):575-80.

31. Jiménez G, Shvartsman SY, Paroush Z. The Capicua repressor--a general sensor of RTK signaling in development and disease. J Cell Sci. 2012;125(6): 1383-91. 
32. LeBlanc VG, Firme M, Song J, Chan SY, Lee MH, Yip S, Chittaranjan S, Marra MA. Comparative transcriptome analysis of isogenic cell line models and primary cancers links capicua (CIC) loss to activation of the MAPK signalling cascade. J Pathol. 2017:242(2):206-20.

33. Da Via MC, Solimando AG, Garitano-Trojaola A, Barrio S, Munawar U, Strifler S, Haertle L, Rhodes N, Teufel E, Vogt C, et al. CIC mutation as a molecular mechanism of acquired resistance to combined BRAF-MEK inhibition in Extramedullary multiple myeloma with central nervous system involvement. Oncologist. 2020;25(2):112-8.

34. Shirazi F, Jones RJ, Singh RK, Zou J, Kuiatse I, Berkova Z, Wang H, Lee HC, Hong S, Dick L, et al. Activating KRAS, NRAS, and BRAF mutants enhance proteasome capacity and reduce endoplasmic reticulum stress in multiple myeloma. Proc Natl Acad Sci U S A. 2020;117(33):20004-14.

35. Huang HF, Xu J, Xu AS, Wan XY, Chen L, Fan FJ, Zhang B, Tang L, Chen WX, Sun $X$, et al. Disturbed MAPK pathway in early bilateral testicular extramedullary relapse of multiple myeloma. Leuk Res. 2020;95:106403.

36. Diaz G, Amicosante M, Jaraquemada D, Butler RH, Guillen MV, Sanchez M, Nombela C, Arroyo J. Functional analysis of HLA-DP polymorphism: a crucial role for DPbeta residues 9, 11, 35, 55, 56, 69 and 84-87 in T cell allorecognition and peptide binding. Int Immunol. 2003;15(5):565-76.

37. Leite FA, Lira RC, Fedatto PF, Antonini SR, Martinelli CE Jr, de Castro M, Neder L, Ramalho LN, Tucci S Jr, Mastelaro MJ, et al. Low expression of HLADRA, HLA-DPA1, and HLA-DPB1 is associated with poor prognosis in pediatric adrenocortical tumors (ACT). Pediatr Blood Cancer. 2014;61(11): 1940-8.

38. Christopher MJ, Petti AA, Rettig MP, Miller CA, Chendamarai E, Duncavage EJ, KIco JM, Helton NM, O'Laughlin M, Fronick CC, et al. Immune escape of relapsed AML cells after allogeneic transplantation. N Engl J Med. 2018; 379(24):2330-41.

39. Meissner M, Konig V, Hrgovic I, Valesky E, Kaufmann R. Human leucocyte antigen class I and class II antigen expression in malignant fibrous histiocytoma, fibrosarcoma and dermatofibrosarcoma protuberans is significantly downregulated. J Eur Acad Dermatol Venereol. 2010;24(11): 1326-32.

40. Hillman GG, Kallinteris NL, Lu X, Wang Y, Wright JL, Li Y, Wu S, Forman JD, Gulfo JV, Humphreys RE, et al. Turning tumor cells in situ into T-helper cellstimulating, MHC class II tumor epitope-presenters: immuno-curing and immuno-consolidation. Cancer Treat Rev. 2004;30(3):281-90.

\section{Publisher's Note}

Springer Nature remains neutral with regard to jurisdictional claims in published maps and institutional affiliations.

Ready to submit your research? Choose BMC and benefit from:

- fast, convenient online submission

- thorough peer review by experienced researchers in your field

- rapid publication on acceptance

- support for research data, including large and complex data types

- gold Open Access which fosters wider collaboration and increased citations

- maximum visibility for your research: over $100 \mathrm{M}$ website views per year

At BMC, research is always in progress.

Learn more biomedcentral.com/submissions 